KEYNOTE SPEECH

\title{
Benefit Considerations of a New Formulation of Kluiklui (Peanut-Cake) Biscuit Supplemented with Breadfruit Flour as Prebiotic Product
}

\author{
Nasser Farid Geraldo \\ Faculty of Agricultural Science and Environment of the Catholic University of West Africa
}

\begin{abstract}
Estimated at about 7.55 billion according to the United Nations (2017) the number of people on earth is growing and this is due to the high birth rate that the world knows. This high number of people on earth will cause problems such as overcrowding, hunger and food insecurity and many others, the most to be feared is food insecurity. Talking about food security is about food, and in trying to address the need for nutritious food, it will be about increasing the productivity of food processing processes and improving practices to ensure a good nutritional status and consequently a good state of health of the populations. In other words, to identify food pathways that can improve the nutritional status of populations. Based on the fact that - The orientation of research towards the exploitation of new sources with high nutritional functionality has led to renewed interest in unconventional crops with potential advantages for local development of populations as well as for industry in recent years - Agricultural development must play a leading role in alleviating hunger and increasing food security locally, regionally and globally (Jones et al., 2011). Agribusiness is a sector that should be stimulated by appropriate technologies, but also and especially by local products valued in order to cope with the increase in food imports, we have been able to identify in the biodiversity from Africa more precisely in the tropical zone the fruits of Artocarpus altilis (Park.) Fosberg, a largely underutilized food energy source (Ferguson 1980, Morton 1990), a fruit that according to Worrell (2002), Orwa (2009), Liu, (2014) and Graham (1981) are highly nutritious, rich in carbohydrates, proteins, fiber and ash consisting of appreciable amounts of calcium, magnesium, potassium and phosphorus, frequently consumed as a starchy staple. Breadfruit has great potential to alleviate hunger and increase food security in the tropics, given the high productivity of the trees of this fruit. And groundnut (Arachis hypogea) is an important source of protein, fats, minerals and vitamins in the diet of rural populations, especially children (Adjou, 2012). Groundnuts play an important role in human nutrition. It is a good source of protein and mineral lipids. These two twinned agricultural products deserve to be the basis of research on food products that can help tackle the problem of food insecurity. And since economic development and its challenges in the agri-food sector, can not overshadow issues related to the cultural identity of a country, because many local foods have so far resisted imported products, thanks to their anchoring in the culture of the populations we also looked at a product produced from the transformation of the peanut commonly called "kluiklui" which distinguishes Benin on the cultural and culinary level. It is a food of choice of certain social classes, which has a very appetizing flavor (Guedou, 2011). In view of all this, we decided to enhance the value of this snack by improving some of its features to find solutions related to several problems such as poverty, malnutrition and many others and the cake biscuit of peanut and breadfruit flour is our end product because biscuit is the most popular food eaten because of its varied taste, long shelf life and relatively low costs, which is similar to in kluiklui. The incorporation of breadfruit flour (Artocarpus altilis (Park.)) Into the formulation may be desirable from a nutritional and healthy point of view. The effect of adding breadfruit flour on the nutritional properties and formation of process contaminants in peanut meal flour biscuits is worth studying. It is therefore in order to make available a product with high nutritional value accessible to all on the one hand and the promotion of groundnut and breadfruit processing that we undertook to carry out our research based on the risks and benefits of a new formulation of kluiklui biscuits incorporated into breadfruit flour as a prebiotic product, because prebiotics are known to stimulate the growth and activity of intestinal bacteria that are potentially useful for health and they also offer improved organoleptic quality and a better balanced nutritional composition. The development and improvement of functional nutritious biscuits, peanut cake recipe in combination with breadfruit flour as a prebiotic product is possible and can help fight against malnutrition which is a public health problem in the countries under development because proteinenergy malnutrition and food insecurity are among the most serious problems facing tropical African countries.
\end{abstract}

